\title{
Chaos in charged Gauss-Bonnet AdS black holes in extended phase space
}

\author{
Sandip Mahish and Chandrasekhar Bhamidipati \\ School of Basic Sciences Indian Institute of Technology Bhubaneswar Jatni, \\ Khurda, Odisha 752050, India
}

(Received 10 March 2019; published 22 May 2019)

\begin{abstract}
We study the onset of chaos due to temporal and spatially periodic perturbations in charged GaussBonnet AdS black holes in extended thermodynamic phase space, by analyzing the zeros of the appropriate Melnikov functions. Temporal perturbations coming from a thermal quench in the unstable spinodal region of P-V diagram may lead to chaos when a certain perturbation parameter $\gamma$ saturates a critical value, involving the Gauss-Bonnet coupling $\alpha$ and the black hole charge $Q$. A general condition following from the equation of state is found, which can rule out the existence of chaos in any black hole. Using this condition, we find that the presence of charge is necessary for chaos under temporal perturbations. In particular, chaos is absent in neutral Gauss-Bonnet and Lovelock black holes in general dimensions. Chaotic behavior continues to exist under spatial perturbations, irrespective of whether the black hole carries a charge or not.
\end{abstract}

DOI: 10.1103/PhysRevD.99.106012

\section{INTRODUCTION}

Black hole solutions and their thermodynamics in general relativity have thrown up remarkable surprises and continue to be an intriguing area of research. In particular, phase transitions of black holes in a variety of backgrounds, such as anti-de Sitter (AdS) space-time have been actively pursued, purely from a gravity point of view and also with holographic motivations in mind [1-9]. More recently, treating the cosmological constant as a dynamical thermodynamical variable (pressure), an extended phase thermodynamics has been proposed, where the first law of black hole mechanics gets modified by a new $p d V$ term [10-21]. The study of PV critical behavior of various black holes confirms the existence of an exact map of black hole small/ large phase transitions to the Van der Waals liquid/gas system [22-26].

It is known that chaos is unavoidable in certain dynamical systems in nature, including black hole physics and cosmology [27-34]. There have been several past works probing chaotic behavior in black holes by various methods, such as, computation of Lyapunov exponents to study stability of orbits, quasinormal modes in ReissnerNordstrom (RN), and Gauss-Bonnet black holes [35,36],

\footnotetext{
*sm19@iitbbs.ac.in

chandrasekhar@iitbbs.ac.in
}

Published by the American Physical Society under the terms of the Creative Commons Attribution 4.0 International license. Further distribution of this work must maintain attribution to the author(s) and the published article's title, journal citation, and DOI. Funded by SCOAP. and Melnikov's [37] method in the context of geodesic motion [27-29]. However, the study of chaos in the context of black hole thermodynamics and phase transitions has only just started emerging [38], partly due to the recent developments where a pressure term in the first law is included, making the connection with Van der Waals system exact [22-26]. In [38], the Melnikov method used in dynamical systems [37-41], developed in the context of Van der Waals system [42], was applied to the case of black holes in extended phase space to extract useful information about the presence of chaos. Temporal and spatial periodic perturbations were introduced in the PV thermodynamic phase space, and the presence of chaos was detected from the study of zeros of Melnikov function. A bound involving the charge of the black hole was also found, beyond which the system becomes chaotic.

In this paper, we take these issues forward by studying chaos in the extended thermodynamic phase of black holes, after incorporating the effects of higher curvature terms in Einstein action. We focus on the case of Gauss-Bonnet(GB) black holes, but the results are also spelled out for Lovelock black holes. Gauss-Bonnet and Lovelock terms are quite important in various contexts such as semiclassical quantum gravity, low energy effective action of string theory, and next to leading order large $\mathrm{N}$ corrections of boundary conformal field theory (CFT) studies in holography [43-62]. They are known to have given interesting insights into the corrections to black hole entropy, viscosity to entropy ratio, and several other recent developments in extended phase space $[49,51-54,56]$. Chaotic dynamics of test objects and instability of certain orbits, in particular, in the context of holography and Gauss-Bonnet theories, has 
also been explored before $[36,63,64]$, however, not from thermodynamic point of view. The extended phase thermodynamics of Gauss-Bonnet black holes in AdS (where the cosmological constant is taken to be dynamical) and its connection to the Van der Waals liquid/gas system via PV criticality is now well studied [57]. Following the study of chaotic behavior for Reissner-Nordstrom black holes in AdS, it is important to know whether the behavior found in [38] is a generic feature of systems exhibiting Van der Waals type phase transitions. With this motivation, we thus study chaotic dynamics in Gauss-Bonnet and other higher derivative theories of gravity, with the inclusion of an additional parameter, such as the Gauss-Bonnet coupling, in addition to the charge (considered in [38]) and find that there appears a new inequality which governs the existence of chaos. We also show that neutral Gauss-Bonnet black holes in five and higher dimensions, in contrast, do not show chaotic behavior under temporal perturbations, despite the fact that a Van der Waals type phase transition and PV criticality exists [57]. We generalize this result to generic black holes systems which have an extended thermodynamic phase space description and starting from the equation of state, we find a new relation which can be used to rule out chaos. However, chaotic behavior under spatial perturbations in the unstable thermodynamic region continues to exist for charged, as well as, neutral black holes. The results are also extended to Lovelock black holes in various dimensions.

The rest of the paper is organized as follows. In Sec. II, we recall the definition of Melnikov function and a few known aspects of thermodynamics of GB black holes in extended phase space formalism. Section III deals with the effect of having a small temporal perturbation in the spinodal region of GB black hole thermodynamic phase space. We first obtain the analogue Hamiltonian system starting from the equation of state of the GB black hole, leading to the determination of homoclinic/heteroclinic orbits. Using the solutions for these orbits, the Melnikov function is computed explicitly and its zeros are analyzed, which give a bound on the parameter $\gamma$ (following from a small temporal perturbation, to be introduced in Sec. III) for the existence of chaotic behavior. This bound is also discussed for Lovelock black holes in higher dimensions, and a general condition for ruling out chaos in any black hole is obtained. In Sec. IV, the effect of a small spatial perturbation leading to the onset of chaos is discussed for GB black holes. Section V contains conclusions.

\section{MELNIKOV'S METHOD AND CHARGED BLACK HOLES IN ADS}

We start in Sec. II A by summarizing the basic technique due to Melnikov for studying the onset of chatotic behavior in Hamiltonian systems. In the following Sec. II B, we collect the main results on charged Gauss-Bonnet black holes in AdS. These are then used for the computation of a

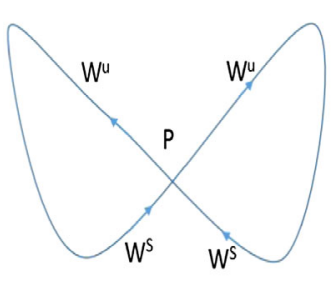

(a)

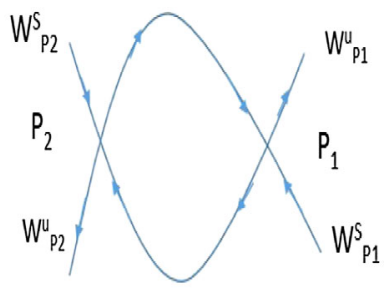

(b)
FIG. 1. (a) Homoclinic orbit. (b) Heteroclinic orbit.

Melnikov function in Secs. III and IV, for studying temporal and spatial perturbations, respectively, in charged Gauss-Bonnet black holes.

\section{A. Melnikov's method for perturbation of Hamiltonian systems}

To understand the Melnikov method, it is useful to start from an evolution equation for a displacement function $x(t)$ as follows:

$$
\dot{x}=f_{0}(x)+\epsilon f_{1}(x, t), \quad x \in \Re^{2 n},
$$

with the following assumptions. First, $\epsilon \ll 1$, corresponding to a small perturbation and the function $f_{1}(x, t)$, is taken to be periodic in $t$. Second, the unperturbed system is Hamiltonian with smooth flow, conserving energy and contains a fixed point which is a homoclinic orbit ${ }^{1}$. There are further nonresonance assumptions on the function $f_{1}(x, t)$, which are necessary for smooth period perturbations and are given in the appropriate sections below. Figure 1 shows sample plots of homoclinic and heteroclinic orbits. For homoclinic orbits, the stable $W^{s}$ and unstable $W^{u}$ manifolds of the saddle connect to each other at the hyperbolic fixed point $P$, while for heteroclinic orbits, the stable manifold of one saddle joins the unstable manifold of the other saddle, as seen in Fig. 1. Now, under a temporal/ spatial perturbation of the system, there will be infinite number of complicated intersection points of stable and unstable manifolds (as it takes an infinite time to approach a saddle point). If the unperturbed homoclinic orbit is considered to be a curve parametrized by time, then at time $t_{0}$, the stable and unstable manifolds are separated by a perpendicular distance (as shown in Fig. 2) given as

$$
d\left(t_{0}\right)=\frac{\epsilon M\left(t_{0}\right)}{\left|\mathbf{f}\left(x_{0}(0)\right)\right|}
$$

Here, $M\left(t_{0}\right)$ is known to be the the Melnikov function, and its explicit form can be shown to be [37-41],

\footnotetext{
${ }^{1}$ The Melnikov method also works for heteroclinic orbits connecting two saddle points and irrespective of whether the solution $x_{0}(t)$ is known analytically or not.
} 


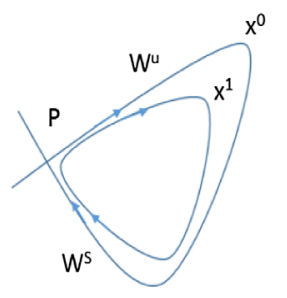

(a)

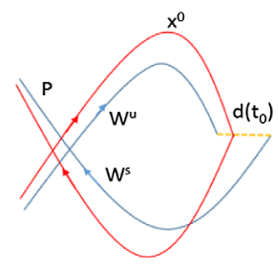

(b)
FIG. 2. Homoclinic orbit: (a) Before perturbation. (b) After perturbation.

$M\left(t_{0}\right)=\int_{-\infty}^{+\infty} \mathrm{f}_{0}^{T}\left(x_{0}\left(t-t_{0}\right)\right) \Omega_{n} \mathrm{f}_{1}\left(x_{0}\left(t-t_{0}\right), t\right) d t$,

with

$\Omega_{n=2}=\left(\begin{array}{cccc}0 & 1 & 0 & 0 \\ -1 & 0 & 0 & 0 \\ 0 & 0 & 0 & 1 \\ 0 & 0 & -1 & 0\end{array}\right)$ and $\Omega_{n=1}=\left(\begin{array}{cc}0 & 1 \\ -1 & 0\end{array}\right)$.

Here, the subscript 1 and 2 stand for the number of degrees of freedom appearing in temporal and spatial perturbations, respectively. Melnikov function, $M\left(t_{0}\right)$, is thus an estimate of the distance $d\left(t_{0}\right)$ for the transverse intersections of stable and unstable orbits. If $M\left(t_{0}\right)$ has a simple zero as a function of $t_{0}$, then for $\epsilon>0$ and for a suitably small value, the stable and unstable manifold of the Hamiltonian system intersect transversally [37-41]. From the SmaleBirkhoff theorem [65], the presence of such intersecting orbits implies that the Poincare map has a invariant hyperbolic set: a Smale horseshoe, which is an indicator of chaos $[41,66]$.

\section{B. Charged Gauss-Bonnet black holes in AdS}

We start with some preliminaries on the thermodynamics of black holes in extended phase space and defining the spinodal region where chaos is found. The EinsteinMaxwell action with a Gauss-Bonnet term and a cosmological constant $\Lambda$, in $d$ dimensions is as follows:

$$
\begin{aligned}
S= & \frac{1}{16 \pi} \int d^{d} x \sqrt{-g}\left[R-2 \Lambda+\alpha_{G B}\left(R_{\mu \nu \gamma \delta} R^{\mu \nu \gamma \delta}\right.\right. \\
& \left.\left.-4 R_{\mu \nu} R^{\mu \nu}+R^{2}\right)-4 \pi F_{\mu \nu} F^{\mu \nu}\right],
\end{aligned}
$$

where $\alpha_{G B}$ is Gauss Bonnet coupling and $\Lambda=-\frac{(d-1)(d-2)}{2 l^{2}}$. $F_{\mu \nu}$ is the Maxwell field strength, defined as $F_{\mu \nu}=$ $\partial_{\mu} A_{\nu}-\partial_{\nu} A_{\mu}$, with the vector potential $A_{\mu}$. Here, we will mostly consider the case with $\alpha_{G B} \geq 0$. The Gauss-Bonnet term, proportional to $\alpha_{G B}$ in the above action, is a topological term in four dimensions, and hence, we take $d \geq 5$. The solution for a static charged GB black hole is given as

$$
d s^{2}=-f(r) d t^{2}+\frac{d r^{2}}{f(r)}+r^{2} d \Omega_{d-2}^{2},
$$

where $d \Omega_{d-2}^{2}$ is a line element of $(d-2)$ dimensional maximally symmetric Einstein space with a volume $\Sigma_{k}$, where $\mathrm{k}$ can be 1,0,-1, corresponding to spherical, Ricci flat, and hyperbolic topology of a black hole horizon, respectively. We will mainly deal with horizon of spherical topology. The general metric function is given by [57]

$$
f(r)=k+\frac{r^{2}}{2 \alpha}\left(1-\sqrt{1+\frac{64 \pi \alpha M}{(d-2) \Sigma_{k} r^{d-1}}-\frac{2 \alpha Q^{2}}{(d-2)(d-3) r^{2 d-4}}-\frac{64 \pi \alpha P}{(d-1)(d-2)}}\right) .
$$

Here, $\alpha=(d-3)(d-4) \alpha_{G B}, M$ and $Q$ are mass and charge of black hole, and the pressure $P=-\frac{\Lambda}{8 \pi}$. Notice that we have considered the cosmological constant to be a thermodynamic variable and replaced it with pressure as is the norm in the extended thermodynamic phase space approach. The equation of state can be written as [57],

$$
P=\frac{d-2}{4 r}\left(1+\frac{2 k \alpha}{r^{2}}\right) T-\frac{(d-2)(d-3) k}{16 \pi r^{2}}-\frac{(d-2)(d-5) k^{2} \alpha}{16 \pi r^{4}}+\frac{Q^{2}}{8 \pi r^{2 d-4}}
$$

Thus, the first law in extended phase space is

$$
d M=T d S+\Phi d Q+V d P+\mathcal{A} d \alpha,
$$

where $S$ is the entropy, $\Phi$ is the electric potential, and $\mathcal{A}$ is conjugate to the GB coupling $\alpha$. The Hawking temperature
$T$ and the thermodynamic volume $V$ are given, respectively, as

$$
T=\frac{1}{4 \pi} f^{\prime}(r)=\frac{16 \pi P r^{4} / 3+2 k r^{2}-\frac{2 Q^{2}}{3 r^{2}}}{4 \pi r\left(r^{2}+2 k \alpha\right)}
$$

and 


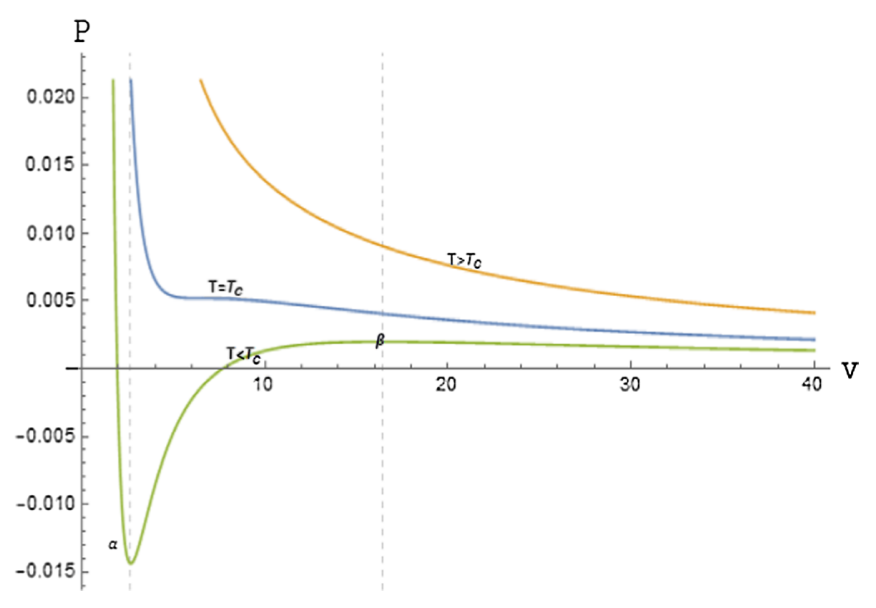

FIG. 3. P-v diagram for the Gauss-Bonnet AdS black hole.

$$
V=\frac{\Sigma_{k} r_{h}^{d-1}}{d-1}
$$

The equation of state in five dimensions is thus,

$$
P=\frac{T}{v}\left(1+\frac{32 \alpha k}{9 v^{2}}\right)-\frac{2 k}{3 \pi v^{2}}+\frac{512 Q^{2}}{729 \pi v^{6}}
$$

where the specific volume $v=\frac{4 r}{d-2}=\frac{4 r}{3}$. The Melnikov method is well suited for studying chaotic behavior in the black hole systems which follow the Van der Waals equation for a phase transition in the extended phase space formalism. To study the behavior of the system in spinodal region, the $P-v$ phase diagram is introduced in Fig. 3, where the labeling of different points is explained below. Denoting $\delta P=\partial P\left(v, T_{0}\right) / \partial v$ and for a temperature $T^{\prime}$ below a critical temperature, the phase space of specific volume, i.e., $v \in[0, \infty]$ ), is divided in to three regimes. $[0, \alpha]$ corresponds to the region where small black holes exist, i.e., the fluid being in liquid phase; i.e., $\delta P<0$. $[\alpha, \beta]$ corresponds to an unstable region, where small and large black hole phase coexists, i.e., $\delta P>0$. The two points $\alpha$ and $\beta$ are determined by $\left.\delta P\right|_{v=\alpha}=\left.\delta P\right|_{v=\beta}=0$. This is the main region of interest in the present case, called the spinodal domain, where a temporal or spatial periodic perturbation leads to chaos under certain conditions, to be discussed below. $[\beta, \infty]$ corresponds to the large black hole domain, i.e., the fluid being vapor and where $\delta P<0$.

\section{TEMPORAL CHAOS IN A SPINODAL REGION}

Here, we study the effect of a small temporally periodic perturbation, when the system is quenched to the unstable spinodal region. We first compute the Hamiltonian for the fluid flow using the black hole equation of state and obtain the Melnikov function, which contains information about the onset of chaos. Let us start by considering a specific volume $v_{0}$ corresponding to an isotherm $T_{0}$, which is fluctuated as follows $[38,42]$ :

$$
T=T_{0}+\epsilon \gamma \cos (\omega t) \cos (X) \quad \text { with } \quad \epsilon \ll 1 .
$$

The fluid flow is assumed to be taking place along the $\mathrm{x}$ axis in a tube of a unit cross section with a fixed volume, which contains a total of a mass $2 \pi / q$ of fluid in a volume $(2 \pi / q) v_{0}$, where $q>0$ is a constant [42]. The fluid is further assumed to be thermoelastic, slightly vicious and isotropic with an additional stress following from the van der Waals-Korteweg theory of capillarity $[42,67,68]$. Here, $X$ represents a column of a black hole of a unit cross section taken between certain points, and the details together with other assumptions are similar to earlier considerations given in $[38,42]$. In the present case, the Hamiltonian is symbolically given to be $[38,42]$,

$$
H=\frac{1}{\pi} \int_{0}^{2 \pi}\left[\frac{u^{2}}{2}+\mathcal{F}(v, T)+\frac{A q^{2}}{2}\left(\frac{\partial v}{\partial X}\right)^{2}\right] d X,
$$

where $u(x, t)=x_{t}(X, t)$ is the velocity of the reference fluid particle and $A$ is a constant. Here,

$$
\mathcal{F}(v, T)=-\int \bar{P}(v, T) d v,
$$

with

$$
\begin{aligned}
\bar{P}(v, T)= & P(v, T) \frac{d V}{d v}=\frac{4 \pi Q^{2}}{9 v^{3}} \\
& \left.+\frac{9}{128} \pi^{2}\left(-6 k v+9 T v^{2}+32 k T \alpha\right)\right) .
\end{aligned}
$$

$\bar{p}(v, T)$ is an effective equation of state obtained by replacing $v$ in terms of the thermodynamic volume $V=$ $\frac{\pi v^{3}}{6}$ before performing the integral. This is important as the Gibbs free energy written in terms of thermodynamic volume remains unchanged during phase transition, and it is the combination $P d V$ that has the right scaling [58,59]. Following the approach in [38,42], ignoring coefficients of order $\mathcal{O}\left(1 / v^{4}\right)$ in Taylor series expansion, the Hamiltonian can be computed to be

$$
\begin{aligned}
H(x, u)= & \frac{u_{1}^{2}+u_{2}^{2}}{2}-\frac{\bar{P}_{v}}{2}\left(v_{0}, T_{0}\right)\left(x_{1}^{2}+x_{2}^{2}\right)-\frac{\bar{P}_{v, v}}{4}\left(v_{0}, T_{0}\right) x_{1}^{2} x_{2} \\
& -\frac{\bar{P}_{v, v, v}}{32}\left(v_{0}, T_{0}\right)\left(x_{1}^{4}+x_{2}^{4}+4 x_{1}^{2} x_{2}^{2}\right) \\
& -\bar{P}_{T}\left(v_{0}, T_{0}\right) \epsilon \gamma \cos (\omega t) x_{1} \\
& -\bar{P}_{v, T}\left(v_{0}, T_{0}\right) \epsilon \gamma \cos (\omega t) x_{1} x_{2} \\
& -\frac{\bar{P}_{v, v, T}}{24}\left(v_{0}, T_{0}\right) 3 \epsilon \gamma \cos (\omega t) x_{1}\left(x_{1}^{2}+2 x_{2}^{2}\right) \\
& +\frac{A q^{2}}{2}\left(x_{1}^{2}+4 x_{2}^{2}\right) .
\end{aligned}
$$


Let us note that the above form of the Hamiltonian is quite generic and is valid for any black hole in extended phase space thermodynamics. For the particular case of charged GB black holes, the relevant Hamiltonian is found to be

$$
\begin{aligned}
H(x, u)= & \frac{u_{1}^{2}+u_{2}^{2}}{2}+\frac{A q^{2}}{2}\left(x_{1}^{2}+4 x_{2}^{2}\right)-\frac{9 \pi^{2}}{128} \epsilon \gamma \cos (\omega t)\left(32 k \alpha+9 v_{0}^{2}\right) x_{1}-\frac{81 \pi^{2}}{128} \epsilon \gamma \cos (\omega t) v_{0} x_{1} x_{2} \\
& -\frac{1}{4}\left(\frac{81 \pi^{2} T_{0}}{64}+\frac{16 \pi Q^{2}}{3 v_{0}^{5}}\right) x_{1}^{2} x_{2}-\frac{1}{2}\left(-\frac{4 \pi Q^{2}}{3 v_{0}^{4}}+\frac{9 \pi}{128}\left(-6 k+18 \pi T_{0} v_{0}\right)\right)\left(x_{1}^{2}+x_{2}^{2}\right) \\
& -\frac{81 \pi^{2}}{512} \epsilon \gamma \cos (\omega t) x_{1}\left(x_{1}+2 x_{2}^{2}\right)+\frac{5 \pi Q^{2}}{6 v_{0}^{6}}\left(x_{1}^{4}+x_{4}+4 x_{1}^{2} x_{2}^{2}\right) .
\end{aligned}
$$

Here, $\left(x_{1}, x_{2}\right)$ and $\left(u_{1}, u_{2}\right)$ are the position and velocities of the first two modes, and the corresponding equations of motion are

$$
\begin{gathered}
\dot{x}_{1}=\frac{\partial H_{2}}{\partial u_{1}}=u_{1}, \\
\dot{x}_{2}=\frac{\partial H_{2}}{\partial u_{2}}=u_{2}, \\
\dot{u}_{1}=-\frac{\partial H_{2}}{\partial x_{1}}-\epsilon \mu_{0} q u_{1}=\frac{9 \pi^{2}}{128} \epsilon \gamma \cos (\omega t)\left(32 k \alpha+9 v_{0}^{2}\right)-A q^{2} x_{1} \\
+\left(-\frac{4 \pi Q^{2}}{3 v_{0}^{4}}+\frac{9 \pi^{2}}{128}\left(-6 k+18 T_{0} v_{0}\right)\right) x_{1}+\frac{81 \pi^{2}}{256} \epsilon \gamma \cos (\omega t) x_{1}^{2} \\
+\frac{81 \pi^{2}}{128} \epsilon \gamma \cos (\omega t) v_{0} x_{2}+\frac{1}{2}\left(\frac{81 \pi^{2} T_{0}}{64}+\frac{16 \pi Q^{2}}{3 v_{0}^{5}}\right) x_{1} x_{2}+\frac{81 \pi^{2}}{512} \epsilon \gamma \cos (\omega t)\left(x_{1}^{2}+2 x_{2}^{2}\right) \\
\quad-\frac{5 \pi Q^{2}}{6 v_{0}^{6}}\left(4 x_{1}^{3}+8 x_{1} x_{2}^{2}\right)-q \epsilon \mu_{0} u_{1}, \\
\dot{u}_{2}=\frac{\partial H_{2}}{\partial x_{2}}-4 \epsilon \mu_{0} q u_{2}=+\frac{81}{128} \pi^{2} \epsilon \gamma \cos (\omega t) v_{0} x_{1}+\frac{1}{4}\left(\frac{81 \pi^{2} T_{0}}{64}+\frac{16 \pi Q^{2}}{3 v_{0}^{5}}\right) x_{1}^{2}-4 A q^{2} x_{2} \\
+\left(-\frac{4 \pi Q^{2}}{3 v_{0}^{4}}+\frac{9 \pi^{2}}{128}\left(-6 k+18 T_{0} v_{0}\right)\right) x_{2}+\frac{81 \pi^{2}}{128} \epsilon \gamma \cos (\omega t) x_{1} x_{2}-\frac{5 \pi Q^{2}}{6 v_{0}^{6}}\left(4 x_{2}^{3}+8 x_{1}^{2} x_{2}\right)-4 q \epsilon \mu_{0} u_{2} .
\end{gathered}
$$

Writing $z=\left(x_{1}, x_{2}, u_{1}, u_{2}\right)^{T}$, Eqs. (3.7)-(3.9) can be written in a compact form as $\dot{z}(t)=f_{0}(z)+\epsilon f_{1}(z, t) ; f_{1}$ is periodic in t, and the unperturbed system with $\epsilon=0$ is given as $\dot{z}(t)=f_{0}(z)$. If we linearize the unperturbed system about $z=0$, we get $\dot{z}_{L}(t)=L z_{L}(t)$. The matrix $L$ can be computed to be $[38,42]$

$$
\mathbf{L}=\left(\begin{array}{cccc}
0 & 0 & 1 & 0 \\
0 & 0 & 0 & 1 \\
-A q^{2}+\psi & 0 & -\epsilon \mu_{0} q & 0 \\
0 & -4 A q^{2}+\psi & 0 & -4 \epsilon \mu_{0} q
\end{array}\right),
$$

with eigen values,

$$
\begin{aligned}
& \lambda_{1,2}=\frac{-\epsilon \mu_{0} q}{2} \pm \frac{1}{2}\left[\epsilon^{2} \mu_{0}^{2} q^{2}-4\left(A q^{2}-\psi\right)\right]^{\frac{1}{2}} \\
& \lambda_{3,4}=-\frac{4 \epsilon \mu_{0} q}{2} \pm\left[4 \epsilon^{2} \mu_{0}^{2} q^{2}-\left(4 A q^{2}-\psi\right)\right]^{\frac{1}{2}}
\end{aligned}
$$

Here,

$$
\psi=-\frac{4 \pi Q^{2}}{3 v_{0}^{4}}+\frac{9 \pi}{128}\left(-6 k+18 \pi T_{0} v_{0}\right) .
$$


Stability of the nodes depends on $q^{2}$. For $q^{2}<\frac{\psi}{A}$, one notes that $\lambda_{1}>0, \lambda_{2}<0$ and both are real; while for $q^{2}>\frac{\psi}{4 A}$, both the eigen values $\lambda_{3,4}=-\frac{4 \epsilon \mu_{0} q}{2} \pm \imath\left[\left(4 A q^{2}-(\psi)\right]^{\frac{1}{2}}\right.$ are imaginary. Regarding $\lambda_{1,2}$, at least one of them has a positive real part and the other a negative real part, which signals a saddle point and an unstable equilibrium of the first node. On the other hand, both $\lambda_{3,4}$ have a negative real part, indicating the existence of a spiral and a stable equilibrium of the second and higher modes [42]. The solution of an unperturbed system [39,42], which is known to exist in the present case for the Hamiltonian given in Eq. (3.6) is

$$
z_{0}(t)=\left(\begin{array}{c}
C_{1} \operatorname{sech}(a t) \\
0 \\
C_{2} \operatorname{sech}(a t) \tanh (a t) \\
0
\end{array}\right),
$$

where

$$
a=\left(\psi-A q^{2}\right)^{\frac{1}{2}}, \quad C_{1}=\frac{a v_{0}^{3}}{2 Q} \sqrt{\frac{3}{5 \pi}} \quad \text { and } \quad C_{2}=-a C_{1} .
$$

Having established the presence of a homoclinic orbit in Eq. (3.11) connecting the origin to itself in the unperturbed system, we now add the small temporal perturbation and compute the Melnikov function defined earlier in Eq. (2.2) to be

$$
\begin{aligned}
M\left(t_{0}\right)= & -\int_{-\infty}^{+\infty}\left[A_{1} \gamma \cos (\omega t) \chi \xi+A_{2} \gamma \cos (\omega t) \xi^{3} \chi\right. \\
& \left.-q \mu_{0} A_{3} \xi^{2} \chi^{2}\right],
\end{aligned}
$$

where $\chi=\operatorname{sech}\left(a\left(t-t_{0}\right)\right)$ and $\xi=\tanh \left(a\left(t-t_{0}\right)\right)$. Further, $A_{1}=\left(\frac{9 \pi^{2} k \alpha}{4}+\frac{81 \pi^{2} v_{0}^{2}}{128}\right) C_{2}, A_{2}=\frac{243 \pi^{2} C_{2} C_{1}^{2}}{512}$, and $A_{3}=C_{2}^{2}$. The evaluation of $M\left(t_{0}\right)$ is best done using the residue theorem, resulting in

$$
M\left(t_{0}\right)=\mathbf{N} \gamma \omega \sin \left(\omega t_{0}\right)-q \mu_{0} \mathbf{I}
$$

where

$$
\mathbf{N}=A_{4} \pi \operatorname{sech}\left(\frac{\pi \omega}{2 a}\right) \quad \text { and } \quad \mathbf{I}=\frac{\pi A_{3}}{2 a},
$$

with

$$
\begin{aligned}
A_{4}= & \frac{C_{2}\left(\frac{81}{128} \pi^{2} v_{0}^{2}+\frac{9}{4} \pi^{2} k \alpha\right)}{a^{2}}+\frac{C_{1}^{2} C_{2}\left(\omega^{2}+a^{2}\right)}{16 a^{4}} \frac{81 \pi^{2}}{64}, \\
& \quad \text { and } A_{3}=C_{2}^{2} .
\end{aligned}
$$

$M\left(t_{0}\right)$ has simple zeros at $\mathbf{N} \gamma \omega \sin \left(\omega t_{0}\right)-q \mu_{0} \mathbf{I}=0$, giving the bound

$$
\left|\frac{q \mu_{0} \mathbf{I}}{\mathbf{N} \gamma \omega}\right| \leq 1
$$

Further, Eq. (3.17) translates into a critical value for the perturbation parameter $\gamma$ of Eq. (3.1), as follows:

$$
\gamma_{\text {critical }}=\left(\frac{\sqrt{3} 512 a^{5} v_{0}^{3} q \cosh \left(\frac{\pi \omega}{2 a}\right) C_{1} \mu_{0}}{18 \sqrt{5} Q \pi^{3 / 2} \omega\left(256 a^{2} k \alpha+9 a^{2} C_{1}^{2}+9 \omega^{2} C_{1}^{2}+72 a^{2} v_{0}^{2}\right)}\right) .
$$

One notes from Eq. (3.18) that a small perturbation with $\gamma>\gamma_{\text {critical }}$ guarantees the transversal intersection of stable and unstable manifolds, including the possible occurrence of Smale horseshoe chaotic motion [41,66]. Chaotic behavior can be noted from Fig. 4, where a numerical plot of time evolution of equations of motion in (3.7)-(3.9) is presented (for simplicity, $x_{2}, u_{2}$ are set to zero). Plots in Figs. 4(a) and 4(b) show normal trajectories of the system in the absence and presence of a small perturbation (but, for $\left.\gamma<\gamma_{\text {critical }}\right)$, respectively. Figure 4(c) shows the onset of chaotic trajectories for $\gamma>\gamma_{\text {critical }}$. The value of $\gamma$ that needs to be chosen for chaotic behavior is shown as the shaded region in the Figs. 5(a) and 5(b), which essentially correspond to the plots of Eq. (3.18). Wherever not mentioned, all the parameters are taken to be unity, without a loss of generality. It is interesting to note from Eq. (3.11), that the homoclinic orbit does not exist for $Q=0$, as the nonlinear term leading to such an orbit is absent from the Hamiltonian in Eq. (3.6). The nonlinear term in the
Hamiltonian in Eq. (3.6) can be traced back to the $\bar{P}_{v, v, v}\left(v_{0}, T_{0}\right)$ term in Eq. (3.5). As seen from Eq. (3.4), this term vanishes for $Q=0$. Thus, we conclude that for neutral Gauss-Bonnet black holes, chaos under temporal perturbations does not occur, unless the black hole carries charge. Noting the importance of the nonvanishing nature of $\bar{P}_{v, v, v}\left(v_{0}, T_{0}\right)$, the above results can be generalized to more general black hole systems by asking: what is the minimum power of $v$ that needs to be present in the equation of state for nonlinearity to appear in the Hamiltonian and lead to chaos? To answer this, let us assume a relation such as $P \propto 1 / v^{n}$, for a generic black hole in extended phase thermodynamics, where $n$ is the largest power of $v$ that occurs in a given black hole equation of state $^{2}$ in a general dimension $d$. The condition to rule out

\footnotetext{
${ }^{2}$ This assumption is valid for most of the static black holes as the equation of state contains polynomials of $v$.
} 


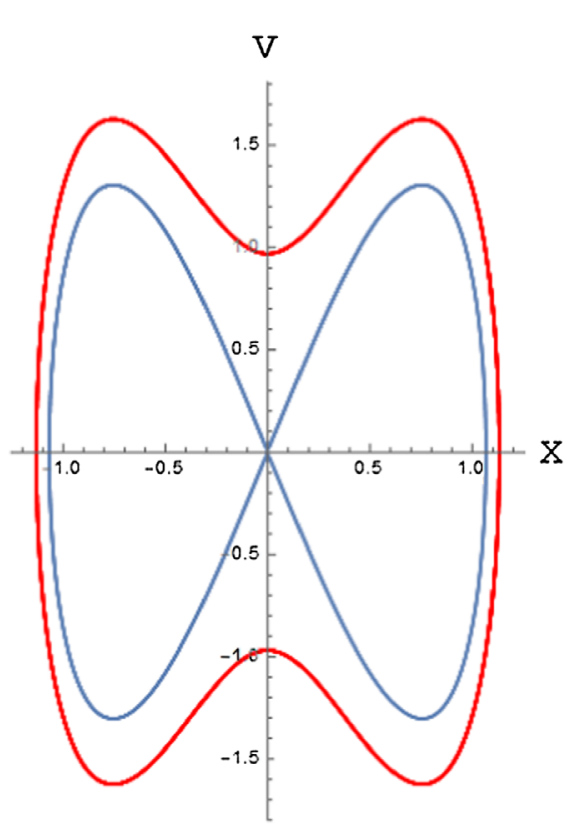

(a)

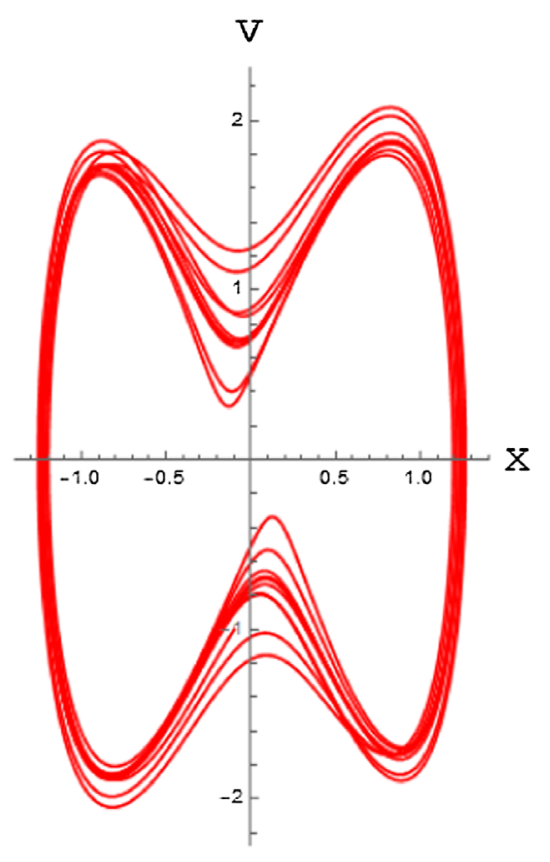

(b)

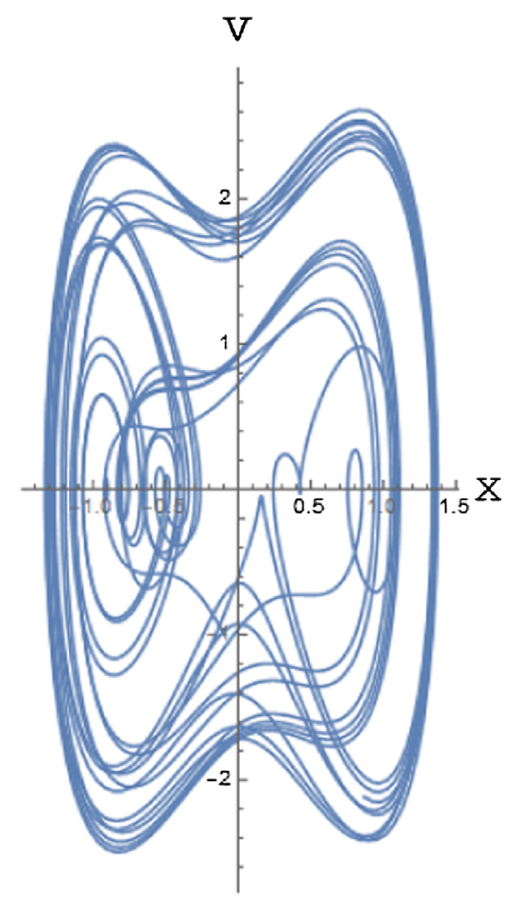

(c)

FIG. 4. Plots show time evolution in the phase space of velocity vs displacement for (a) $\epsilon=0$, (b) $\epsilon \neq 0, \gamma<\gamma_{\text {critical }}$, (c) $\epsilon \neq 0$, $\gamma>\gamma_{\text {critical }}$.

nonlinearity in the Hamiltonian in Eq. (3.5) and the absence of chaotic behavior is that $\bar{P}_{v, v, v}\left(v_{0}, T_{0}\right)=0$. Solving this equation, we get a relation between $n$ and $d$ as

$d=2, n>0, \quad d>i, n=d-i \quad$ for $i=3,4,5$.

Let us note that the conditions in Eq. (3.19) are obtained for any generic black hole in AdS with extended phase

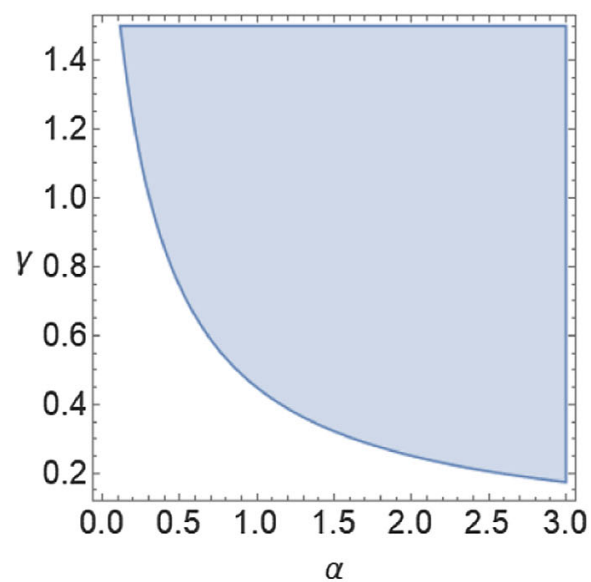

(a) thermodynamic description and can be used to rule out chaos based on the equation of state itself. For instance, as seen from Eq. (2.7), the largest power of $v$ in the equation of state for a neutral Gauss-Bonnet black hole in a general dimension $d$ is $n=4$. Either one of the conditions, given in Eq. (3.19), is always satisfied for any $d>4$ for $n=4$. We thus conclude that chaotic behavior under temporal perturbations would be absent for neutral Gauss-Bonnet black holes in any dimension. On the other hand, for charged GB

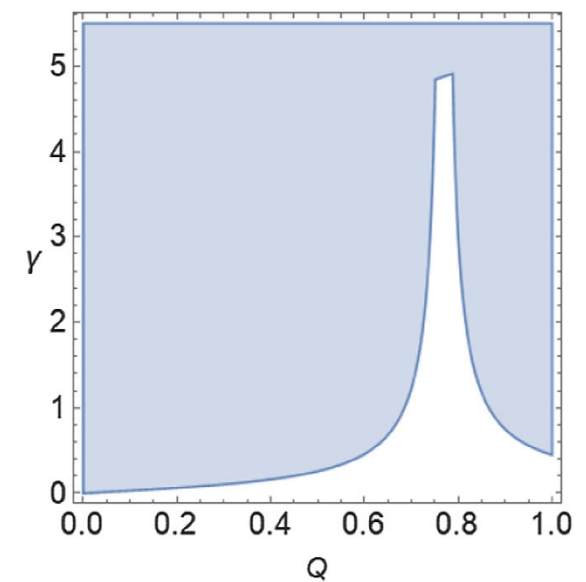

(b)

FIG. 5. Shaded region denotes the onset of chaotic motion: $v_{0}=2.75, T_{0}=0.01$ (a) $\gamma$ vs $\alpha$ plot for charged GB black holes in $d=5$, for $Q=1$, (b) $\gamma$ vs $Q$ plot for charged GB black holes in $d=5$ for $\alpha=1$. 
black holes, there is a term in the equation of state in Eq. (2.7), which contains higher powers of $v$, and it can be checked that the conditions in Eq. (3.19) are not satisfied in any dimension. Thus, chaotic behavior is possible, once the perturbation parameters satisfy the constraints put forward in Eq. (3.18). These differences between neutral and charged black holes should be investigated further for better understanding, especially, because the spinodal region in the PV diagram continues to exist, irrespective of the presence of charge $Q$ or not.

We now extend the results obtained above to the more general case of Lovelock black holes in higher dimensions and check for chaotic behavior. The details of the action and PV critical behavior are discussed in detail in $[61,62]$, and we only need to recall the equation of state given as

$$
P=\frac{T}{v}+\frac{32 k \alpha T}{(d-2)^{2} v^{3}}+\frac{256 k^{2} T \alpha^{2}}{(d-2)^{4} v^{5}}-\frac{(d-3) k}{(d-2) \pi v^{2}}-\frac{16 k^{2}(d-5) \alpha}{(d-2)^{3} \pi v^{4}}-\frac{256 k^{3}(d-7) \alpha^{2}}{3(d-2)^{5} \pi v^{6}}+\frac{16^{d-3}(d-3) Q^{2}}{\pi(d-2)^{(2 d-5)} v^{(2 d-4)}}
$$

It is known that a Van der Waals type phase transition exists in these theories, together with a presence of a spinodal unstable region. The procedure discussed in this section can be straightforwardly extended to the present case in all dimensions, starting from the equation of state in Eq. (3.20) in general dimensions. Applying the condition in Eq. (3.19) to the equation of state in Eq. (3.20) above, chaos can be ruled out for neutral third order Lovelock black holes starting from the dimension $d=7$. For the charged case, however, the nonlinear terms following from the relevant Hamiltonian in Eq. (3.5) will be present, and we see below that chaotic behavior above a certain value of $\gamma$ persists. We have computed analytically the expressions for the Hamiltonian, Melnikov functions, and associated bound on $\gamma$, but they are cumbersome and otherwise not very illuminating. We suppress the expressions and present a plot of $\gamma$ vs $\alpha$ and $Q$ in seven dimensions in Fig. 6, where the shaded parts show the allowed regions of $\gamma$ for which temporal chaos will be present.

Now, let us comment on the chaotic behavior in the case of RN AdS black holes in general dimensions. In this case, the equation of state is given as [69]

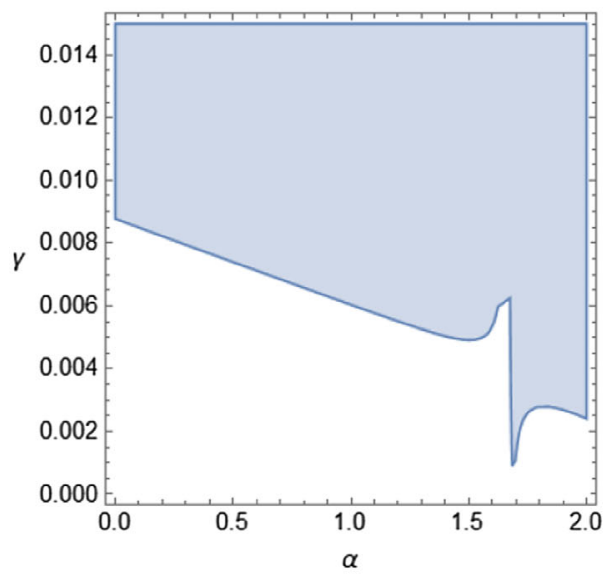

(a)

$$
P=\frac{T}{v}-\frac{d-3}{(d-2) \pi v^{2}}+\frac{(d-3) 2^{4(d-3)} Q^{2}}{(d-2)^{2 d-5} \pi v^{2(d-2)}}
$$

It can be checked explicitly, that none of the conditions in Eq. (3.19) are satisfied in any dimension, and hence, chaos will exist beyond a certain value of the perturbation parameter $\gamma$ in RN AdS black holes in any dimension. As the Gauss-Bonnet terms are total derivative terms in four dimensions, we can start comparing with the RN AdS case (by setting $\alpha=0$ ), starting from five dimensions. In fact, setting $\alpha=0$ in Eq. (3.18), gives the limit on $\gamma$, beyond which chaos will exist in RN AdS black holes in five dimensions. The corresponding result in four dimensions was explicitly computed in [38]. Thus, the conclusion that chaotic behavior should be present in the four dimensional example of RN AdS black holes, studied in [38], is in conformity with our general condition in Eq. (3.19). Furthermore, as $\alpha$ increases, it can be noted from Fig. 5(a), that the GB system becomes chaotic for even smaller values of the perturbation parameter $\gamma$.

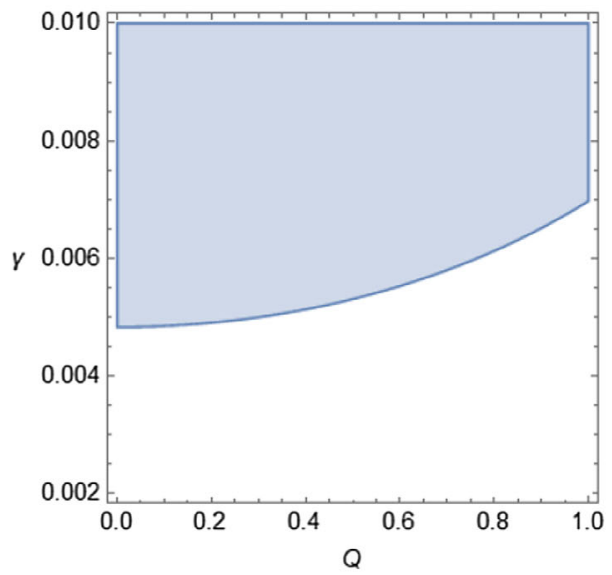

(b)

FIG. 6. Charged Lovelock black holes in $d=7$, with $v_{0}=1.44, T_{0}=0.1$ : Plots of (a) $\gamma$ vs $\alpha$ for $Q=2$ and (b) $\gamma$ vs $Q$ for $\alpha=1$. Values of $\gamma$ in the shaded region lead to onset of chaotic motion. 


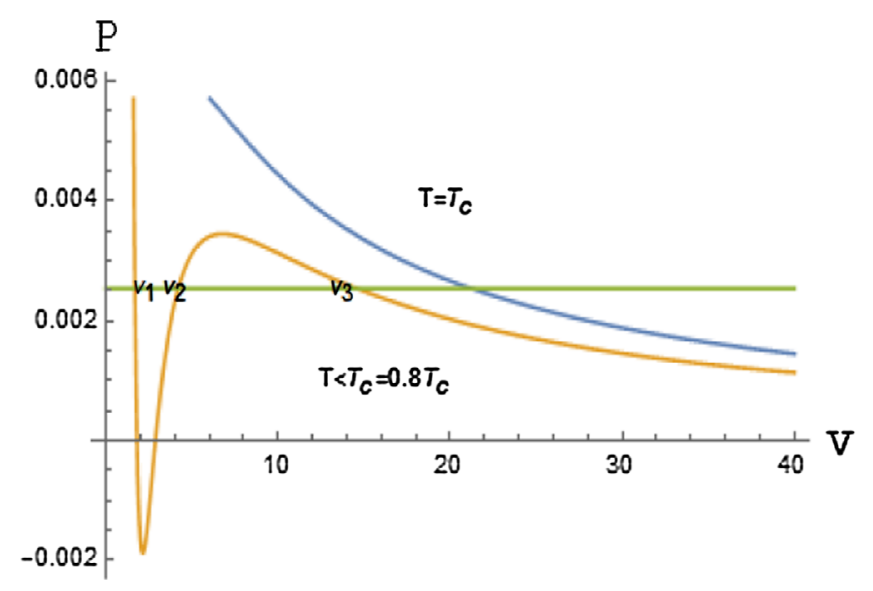

FIG. 7. Charged GB Maxwell equal area construction for $Q=1, \alpha=1, k=1, T=0.8 T_{c}$.

To conclude this section, we note that the presence of charge $Q$ is necessary for triggering chaos under temporal perturbations in the extended thermodynamic phase space of black hole systems.

\section{SPATIAL PERTURBATIONS AND CHAOS}

In this section, our aim is to study the effect of a small spatially periodic perturbation in the equilibrium state solutions about a subcritical temperature given as follows [42]:

$$
T=T_{0}+\epsilon \cos (q x) .
$$

Korteweig's theory gives the Piola stress tensor as [42]

$$
\tau=-P(v, T)-A v^{\prime \prime},
$$

where ' stands for $\frac{d}{d x} \cdot P(v, T)$ is supplied by the GB black hole equation of state from Eq. (2.11) and $T$ is absolute

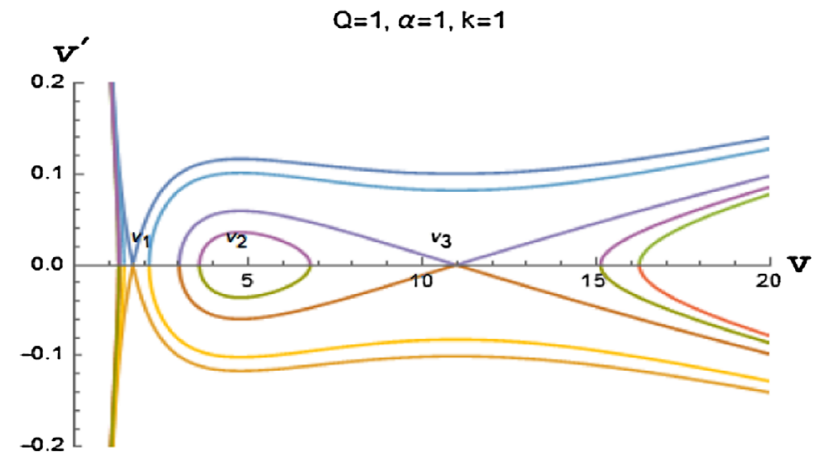

(a) temperature with $A>0$. For a zero body force balance of linear momentum, one sets $\tau^{\prime}=0$, giving $\tau=B=$ constant. Thus, $B$ is the ambient pressure as $|x| \rightarrow \infty$; using this, Eq. (4.2) yields

$$
v^{\prime \prime}+P(v, T)=B .
$$

Let us start by discussing the unperturbed system, where one starts by setting $T=T_{0}$ in Eq. (4.3). The fixed points of the system in Eq. (4.3) can be found, which are the specific volumes corresponding to ambient pressures B for different given temperatures. We choose a set of sample temperatures, $0.8 T_{c}$ and $0.7 T_{C}$, and call the corresponding fixed points as $\left(v_{1}, v_{2}, v_{3}\right)$ and $\left(w_{1}, w_{2}, w_{3}\right)$. For the case of $T_{0}=0.8 T_{c}$, these are shown in Fig. 7, with an analogous construction assumed at $T_{0}=0.7 T_{c}$. Let us note that the Maxwell equal area construction for Gauss Bonnet black holes done in [60] is useful while plotting Fig. 7. Now, from Eq. (4.3) and Fig. 7, one infers three different kinds of orbits in the $v^{\prime}-v$ phase plane.

(i) Case-1: In this case, we choose the pressure in the range $P\left(v_{1}, T_{0}\right)<B<P\left(\beta, T_{0}\right)$ and get a homoclinic orbit connecting a saddle point $v_{3}$ to itself. Corresponding phase orbits are shown in Figs. 8(a) and 8(b) for charged and neutral black holes, respectively.

(ii) Case-2: Choosing the pressure in the range $P\left(\alpha, T_{0}\right)<B<P\left(v_{2}, T_{0}\right)$ results in a homoclinic orbit connecting a saddle point $v_{1}$ to itself, as in case- 1 above. Corresponding phase orbits are presented in Figs. 9(a) and 9(b) for charged and neutral black holes, respectively.

(iii) Case-3: In this case, the pressure is taken such that $P\left(v_{1}, T_{0}\right)=B=P\left(v_{2}, T_{0}\right)$; this results in a heteroclinic orbit connecting $v_{1}$ with $v_{3}$. Corresponding phase orbits are shown in Figs. 10(a) and 10(b) for charged and neutral black holes, respectively.

Including a small spatial perturbation given in Eq. (4.1), we can rewrite the Eq. (4.3) for perturbed system as follows:

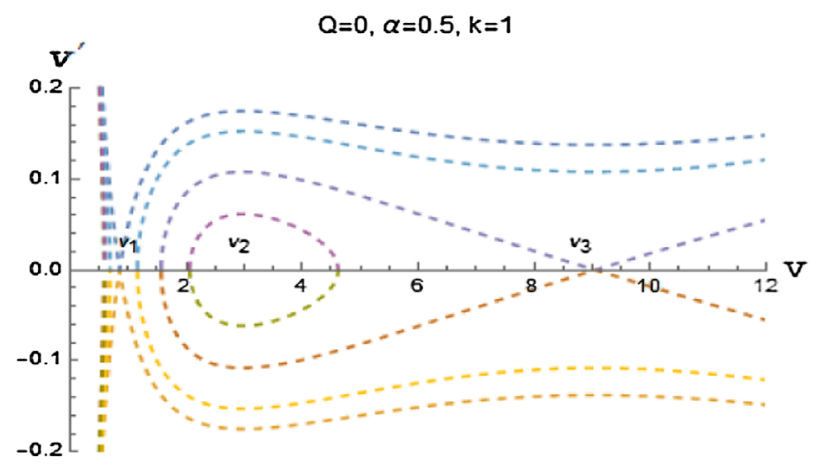

(b)

FIG. 8. Case1: (a) Charged Gauss-Bonnet with $v_{1}=1.68107, v_{2}=4.77519, v_{3}=10.9746$. (b) Neutral Gauss-Bonnet with $v_{1}=0.849379, v_{2}=2.97856, v_{3}=9.04772$. 


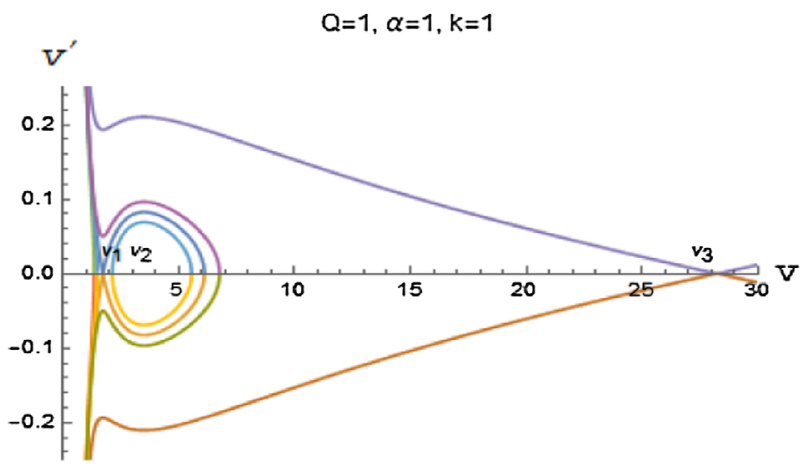

(a)

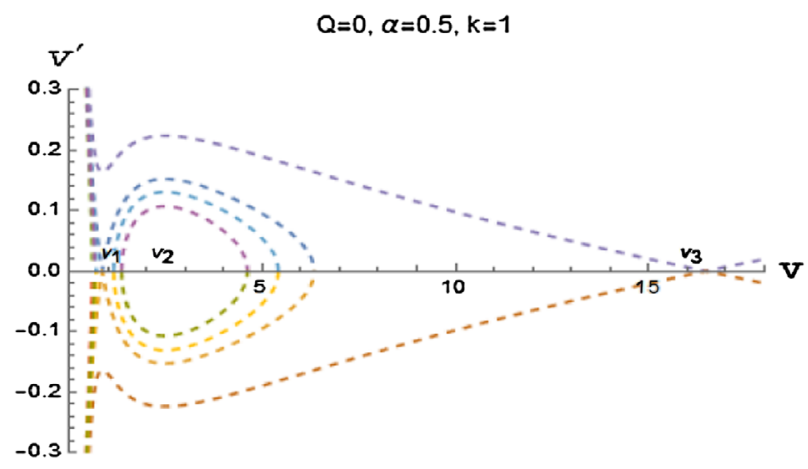

(b)

FIG. 9. Case2: (a) Charged Gauss-Bonnet with $v_{1}=1.73389, v_{2}=3.51509, v_{3}=28.2263$. (b) Neutral Gauss-Bonnet with $v_{1}=0.862346, v_{2}=2.47978, v_{3}=16.4746$.

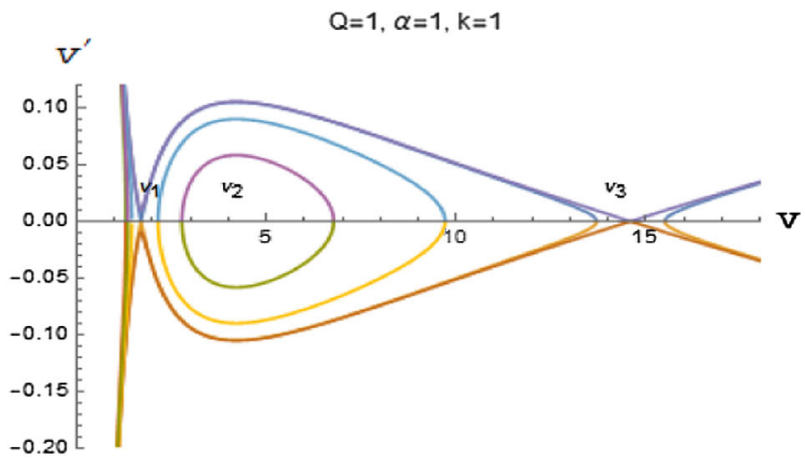

(a)

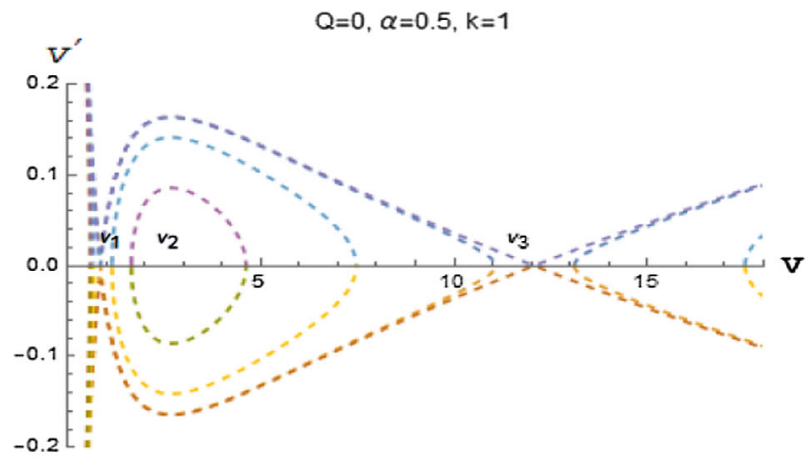

(b)

FIG. 10. Case3: (a) Charged Gauss-Bonnet with $v_{1}=1.69635, v_{2}=4.20704, v_{3}=14.593$. (b) Neutral Gauss-Bonnet with $v_{1}=0.855689, v_{2}=2.68773, v_{3}=12.0659$.

$$
v^{\prime \prime}=B-P\left(v, T_{0}\right)-\frac{\epsilon \cos (q x)}{v} .
$$

Melnikov function from Eq. (2.2) written suitably for spatially perturbed systems is

$M\left(x_{0}\right)=\int_{-\infty}^{\infty} f\left(z\left(x-x_{0}\right)\right) \Omega_{n=1} g\left(z\left(x-x_{0}\right), x\right) d x$.

Setting $v^{\prime}=h$, Eq. (4.4) converts to a set of first order equations as

$$
\begin{aligned}
v^{\prime} & =h \\
h^{\prime} & =B-P\left(v, T_{0}\right)-\frac{\epsilon \cos (q x)}{v} .
\end{aligned}
$$

As in the previous section, writing general solutions for (homoclinic or heteroclinic) orbit as

$$
z(x)=\left(\begin{array}{c}
v_{0}\left(x-x_{0}\right) \\
h_{0}\left(x-x_{0}\right),
\end{array}\right)
$$

and using them in Eq. (4.4), one can write $f\left(z\left(x-X_{0}\right)\right)$ and $g\left(z\left(x-x_{0}\right), x\right)$ as

$$
f\left(z\left(x-X_{0}\right)\right)=\left(\begin{array}{c}
h_{0}\left(x-x_{0}\right) \\
B-P\left(v_{0}\left(x-x_{0}\right), T_{0}\right)
\end{array}\right),
$$

$g\left(z\left(x-X_{0}\right), x\right)=\left(\begin{array}{c}0 \\ -\frac{\cos (q, x)}{v_{0}\left(x-x_{0}\right)}\end{array}\right)$.

Using these in Eq. (4.5), Melnikov function is finally

$$
M\left(x_{0}\right)=-\int_{-\infty}^{+\infty} \frac{h_{0}\left(x-x_{0}\right) \cos (q x)}{v_{0}\left(x-x_{0}\right)} d x .
$$

Changing variables to $R=x-x_{0}$, the Melnikov function becomes

$$
M\left(x_{0}\right)=-L \cos \left(q x_{0}\right)+W \sin \left(q x_{0}\right)
$$

with

$$
\begin{aligned}
L & =\int_{-\infty}^{\infty} \frac{h_{0}(R) \cos (q R)}{v_{0}(R)} d R, \\
W & =\int_{-\infty}^{\infty} \frac{h_{0}(R) \sin (q R)}{v_{0}(R)} d R .
\end{aligned}
$$

From the structure of the Melnikov function and following the arguments in [42], $M\left(x_{0}\right)$ always possesses simple 
zeros, signaling chaos. The results of this section can be carried over to charged Lovelock black holes in various dimensions, and we have checked that the general features found in this section continue to exist, namely the system exhibits homoclinic orbits for the cases- 1 and 2 discussed above; for case-3, the system has homoclinic as well as heteroclinic orbits. The presence of chaos under spatial perturbations is found in all three cases in Lovelock black holes in higher dimensions, irrespective of whether the charge is present or not, unlike the case of temporal perturbations discussed in last section.

\section{CONCLUSIONS}

In this work, we studied the emergence of chaotic behavior under temporal and spatial perturbations in the spinodal region of charged and neutral Gauss-Bonnet black holes in extended thermodynamic phase space. The perturbed Hamiltonian system corresponding to the motion of the fluid in the spinodal region, following from the black hole equation of state, was obtained and shown to possess nonlinear terms giving homoclinic/heteroclinic orbits in phase space. Analysis of the zeroes of the appropriate Melnikov functions gives information about the onset of chaos in the thermodynamic phase space. As regards to temporal perturbations, chaotic behavior is found to be present in charged GB black holes in five dimensions. The zeros of the Melnikov function give a bound on the perturbation parameter for chaos to exist. This was computed analytically, such as the one in Eq. (3.18) and depends on the charge $Q$ and the GB coupling $\alpha$. It is important to note that in this paper, the computations were performed explicitly in five dimensions, as the GaussBonnet term is a total derivative in four dimensions and does not effect the black hole solution. Setting the GB coupling $\alpha=0$ in Eq. (3.18), gives us the bound on $\gamma$ for chaos to exist, for the corresponding RN AdS black holes in five dimensions. These general conclusions hold true in any dimension, based on the condition in Eq. (3.19). For instance, based on Eqs. (3.19) and (3.21), it can be said that in four dimensions, chaotic behavior should exist beyond a certain value of $\gamma$. This result is in conformity with the analysis in [38], where the explicit value of $\gamma$ was computed in four dimensions. When $\alpha$ is nonzero, we find that the onset of chaotic behavior occurs for even lower values of the temporal perturbation parameter $\gamma$, showing the sensitivity of chaotic behavior in the presence of GB terms. Intriguingly, the chaotic behavior under temporal perturbations is not present for neutral GB and Lovelock black holes in general dimensions, which needs to be investigated further. A general condition was derived in Eq. (3.19), which can be used to rule out chaos under temporal perturbations in general dimensions by analyzing the equation of state provided by the black hole. In every dimension, for chaos to exist, the highest power of $v$ in the equation of state cannot be lower than a certain value [as governed by Eq. (3.19)]. The reason for existence of chaotic behavior in charged black holes is that the term dependent on the charge $Q$ in the black hole equation of state, increases quadratically with $v$, as the number of dimensions increases. This can be seen from the last term in the GB and RN AdS equations of state given in Eqs. (2.7) and (3.21), respectively. Thus, chaotic behavior crucially depends on the number of dimensions as well as the equation of state of the black hole.

Under spatial perturbations, the existence of homoclinic and heteroclinic orbits is found to exist in charged as well as neutral GB black holes, and the phase space plots were given. The extension of results to Lovelock black holes in higher dimensions was discussed. It would be interesting to understand the holographic aspects of these chaotic behaviors, particularly found in the unstable small/large black hole phase transition domain, not just in GB black holes, but also in other charged and neutral black holes in AdS, considering other stringy corrections. More importantly, the absence of chaos in neutral black holes needs to be understood better.

\section{ACKNOWLEDGMENTS}

The authors would like to thank the anonymous referee for helpful suggestions. S. M. thanks IIT Bhubaneswar for Institute Research Fellowship.
[1] J. D. Bekenstein, Black holes and entropy Phys. Rev. D, 7, 2333, 1973.

[2] J. D. Bekenstein, Generalized second law of thermodynamics in black-hole physics, Phys. Rev. D, 9, 3292, 1974.

[3] S. W. Hawking, Particle creation by black holes, Commun. Math. Phys. 43, 199 (1975) Erratum, Commun. Math. Phys. 46, 206(E) (1976).
[4] S. W. Hawking, Black holes and thermodynamics, Phys. Rev. D 13, 191 (1976).

[5] G. W. Gibbons and S. W. Hawking, Action integrals and partition functions in quantum gravity, Phys. Rev. D 15, 2752 (1977).

[6] S. W. Hawking and D. N. Page, Thermodynamics of black holes in anti-De Sitter space, Commun. Math. Phys. 87, 577 (1983). 
[7] J. M. Maldacena, The Large N limit of superconformal field theories and supergravity, Int. J. Theor. Phys. 38, 1113 (1999) Adv. Theor. Math. Phys. 2, 231 (1998).

[8] E. Witten, Anti-de Sitter space and holography, Adv. Theor. Math. Phys. 2, 253 (1998).

[9] S. S. Gubser, I. R. Klebanov, and A. M. Polyakov, Gauge theory correlators from noncritical string theory, Phys. Lett. B 428, 105 (1998).

[10] D. Kastor, S. Ray, and J. Traschen, Enthalpy and the mechanics of AdS black holes, Classical Quantum Gravity 26, 195011 (2009).

[11] M. M. Caldarelli, G. Cognola, and D. Klemm, Thermodynamics of Kerr-Newman-AdS black holes and conformal field theories, Classical Quantum Gravity 17, 399 (2000).

[12] S. Wang, S.-Q. Wu, F. Xie, and L. Dan, The First laws of thermodynamics of the $(2+1)$-dimensional BTZ black holes and Kerr-de Sitter spacetimes, Chin. Phys. Lett. 23, 1096 (2006).

[13] Y. Sekiwa, Thermodynamics of de Sitter black holes: Thermal cosmological constant, Phys. Rev. D73, 084009 (2006).

[14] E. A. Larranaga Rubio, Stringy generalization of the first law of thermodynamics for rotating BTZ black hole with a cosmological constant as state parameter, arXiv: 0711.0012.

[15] B. P. Dolan, The cosmological constant and the black hole equation of state, Classical Quantum Gravity 28, 125020 (2011).

[16] M. Cvetic, G. Gibbons, D. Kubiznak, and C. Pope, Black hole enthalpy and an entropy inequality for the thermodynamic volume, Phys. Rev. D84, 024037 (2011).

[17] B. P. Dolan, Pressure and volume in the first law of black hole thermodynamics, Classical Quantum Gravity 28, 235017 (2011).

[18] M. Henneaux and C. Teitelboim, The cosmological constant as a canonical variable, Phys. Lett. B 143, 415 (1984).

[19] C. Teitelboim, The cosmological constant as a thermodynamic black hole parameter, Phys. Lett. B 158, 293 (1985).

[20] M. Henneaux and C. Teitelboim, The cosmological constant and general covariance, Phys. Lett. B 222, 195 (1989).

[21] C. V. Johnson and F. Rosso, Holographic heat engines, entanglement entropy, and renormalization group flow, Classical Quantum Gravity 36, 015019 (2019).

[22] A. Chamblin, R. Emparan, C. V. Johnson, and R. C. Myers, Charged AdS black holes and catastrophic holography, Phys. Rev. D 60, 064018 (1999); Holography, thermodynamics, and fluctuations of charged AdS black holes, Phys. Rev. D 60104026 (1999).

[23] A. Chamblin, R. Emparan, C. V. Johnson, and R. C. Myers, Holography, thermodynamics and fluctuations of charged AdS black holes, Phys. Rev. D 60, 104026 (1999).

[24] D. Kubiznak and R. B. Maan, P-V criticality of charged AdS black holes, J. High Energy Phys. 07 (2012) 033.

[25] D. Kubiznak, R. B. Mann, and M. Teo, Black hole chemistry: thermodynamics with Lambda, Classical Quantum Gravity 34, 063001 (2017).

[26] S. Gunasekaran, R. B. Mann, and D. Kubiznak, Extended phase space thermodynamics for charged and rotating black holes and Born-Infeld vacuum polarization, J. High Energy Phys. 11 (2012) 110.

[27] L. Bombellitf and E. Calzetta, Chaos around a black hole, Classical Quantum Gravity 9, 2573 (1992).

[28] P. S. Letelier and W. M. Vieira, Chaos in black holes surrounded by gravitational waves, Classical Quantum Gravity 14, 1249 (1997).

[29] M. Santoprete and G. Cicogna, Chaos in black holes surrounded by electromagnetic fields, Gen. Relativ. Gravit. 34, 1107 (2002).

[30] G. A. Monerat, H. P. de Oliveira, and I. D. Soares, Chaos in preinflationary Friedmann-Robertson-Walker universes, Phys. Rev. D 58, 063504 (1998).

[31] G. N. Felder and L. Kofman, Nonlinear inflaton fragmentation after preheating, Phys. Rev. D 75, 043518 (2007).

[32] F. L. Dubeibe, L. A. Pachon, and J. D. Sanabria-Gomez, Chaotic dynamics around astrophysical objects with nonisotropic stresses, Phys. Rev. D 75, 023008 (2007).

[33] J. R. Gair, C. Li, and I. Mandel, Observable properties of orbits in exact bumpy spacetimes, Phys. Rev. D 77, 024035 (2008).

[34] S. Chen, M. Wang, and J. Jing, Chaotic motion of particles in the accelerating and rotating black holes spacetime, J. High Energy Phys. 09 (2016) 082.

[35] V. Cardoso, A. S. Miranda, E. Berti, H. Witek, and V. T. Zanchin, Geodesic stability, Lyapunov exponents and quasinormal modes, Phys. Rev. D 79, 064016 (2009).

[36] R. A. Konoplya and Z. Stuchlík, Are eikonal quasinormal modes linked to the unstable circular null geodesics?, Phys. Lett. B 771, 597 (2017).

[37] V. K. Melnikov, On the stability of the center for time periodic perturbations, Trans. Moscow Math. Soc. 121 (1963); J. Guckenheimer and P. J Holmes, Nonlinear Oscillations, Dynamical Systems, and Bifurcations of Vector Fields, Applied Math. Sciences. Vol. 42 (SpringerVerlag, New York, 1983).

[38] M. Chabab, H. El Moumni, S. Iraoui, K. Masmar, and S. Zhizeh, Chaos in charged AdS black hole extended phase space, Phys. Lett. B 781, 316 (2018).

[39] P. Holmes, A nonlinear oscillator with a strange attractor, Phil. Trans. Roy. Sot. A 292, 419 (1979).

[40] P. Holmes, A partial differential equation with infinitely many periodic orbits: Chaotic oscillations of a forced beam, Arch. Ration. Mech. Anal. 76, 135 (1981).

[41] P. Holmes, Poincare celestial mechanics, dynamicalsystems theory and chaos, Phys. Rep. 193, 137 (1990).

[42] M. Slemrod, Temporal and spatial chaos in a van der Waals fluid due to periodic thermal fluctuations, Advances Applied Math. D 6, 135 (1985).

[43] D. Lovelock, The Einstein tensor and its generalizations, J. Math. Phys. 12, 498 (1971).

[44] B. Zwiebach, Curvature squared terms and string theories, Phys. Lett. 156B, 315 (1985).

[45] D. G. Boulware and S. Deser, String Generated Gravity Models, Phys. Rev. Lett. 55, 2656 (1985).

[46] D. L. Wiltshire, Spherically symmetric solutions of Einstein-Maxwell theory with a Gauss-Bonnet term, Phys. Lett. 169B, 36 (1986).

[47] R. C. Myers and J. Z. Simon, Black hole thermodynamics in Lovelock gravity, Phys. Rev. D 38, 2434 (1988). 
[48] D. L. Wiltshire, Black holes in string generated gravity models, Phys. Rev. D 38, 2445 (1988).

[49] S. Nojiri and S. D. Odintsov, Anti-de Sitter black hole thermodynamics in higher derivative gravity and new confining deconfining phases in dual CFT, Phys. Lett. B 521, 87 (2001) Erratum, Phys. Lett. B 542, 301(E) (2002).

[50] R. G. Cai, Gauss-Bonnet black holes in AdS spaces, Phys. Rev. D 65, 084014 (2002).

[51] A. Sen, Entropy function for heterotic black holes, J. High Energy Phys. 03 (2006) 008.

[52] M. Brigante, H. Liu, R. C. Myers, S. Shenker, and S. Yaida, Viscosity bound violation in higher derivative gravity, Phys. Rev. D 77, 126006 (2008).

[53] R. G. Cai, Z. Y. Nie, N. Ohta, and Y. W. Sun, Shear viscosity from Gauss-Bonnet gravity with a dilaton coupling, Phys. Rev. D 79, 066004 (2009).

[54] C. V. Johnson, Gauss-Bonnet black holes and holographic heat engines beyond large $N$, Classical Quantum Gravity 33, 215009 (2016).

[55] M. M. Qaemmaqami, Criticality in third order lovelock gravity and butterfly effect, Eur. Phys. J. C 78, 47 (2018).

[56] C. Bhamidipati and P. K. Yerra, A note on Gauss-Bonnet black holes at criticality, Phys. Lett. B 772, 800 (2017).

[57] R. G. Cai, L. M. Cao, L. Li, and R. Q. Yang, P-V criticality in the extended phase space of Gauss-Bonnet black holes in AdS space, J. High Energy Phys. 09 (2013) 005.

[58] S. Q. Lan, J. X. Mo, and W. B. Liu, A note on Maxwell's equal area law for black hole phase transition, Eur. Phys. J. C 75, 419 (2015).

[59] H. Xu and Z. M. Xu, Maxwell's equal area law for Lovelock thermodynamics, Int. J. Mod. Phys. D 26, 1750037 (2017).
[60] A. Belhaj, M. Chabab, H. El moumni, K. Masmar, and M. B. Sedra, Maxwell's equal-area law for Gauss-BonnetAnti-de Sitter black holes, Eur. Phys. J. C 75, 71 (2015).

[61] M. H. Dehghani, N. Alinejadi, and S. H. Hendi, Topological black holes in Lovelock-Born-Infeld gravity, Phys. Rev. D 77, 104025 (2008).

[62] J. X. Mo and W. B. Liu, $P-V$ criticality of topological black holes in Lovelock-Born-Infeld gravity, Eur. Phys. J. C 74, 2836 (2014).

[63] E. J. Kim and S. Kawai, Chaotic dynamics of the Bianchi IX universe in Gauss-Bonnet gravity, Phys. Rev. D 87, 083517 (2013).

[64] D. Z. Ma, J. P. Wu, and J. Zhang, Chaos from the ring string in a Gauss-Bonnet black hole in AdS5 space, Phys. Rev. D 89, 086011 (2014).

[65] G. D. Birkhoff, Nouvelles recherches sur les systemes dynamiques, Collected Mathematical Papers Vol. 2 (American Mathematical Society, Providence, RL, 1950), pp. 530-662; S. Smale, Diffeomorphisms with many periodic points, Differential and Combinatorial Topology (Princeton University Press, Princeton, NJ, 1965), pp. 63-80.

[66] S. Smale, Diffeomorphisms with many periodic points, Matematika 11, 88 (1967); S. S. Cairns, Differential and Combinatorial Topology (A Symposium in Honor of Marston Morse) (1965), pp. 63-80.

[67] B. U. Felderhof, Dynamics of the diffuse gas-liquid interface near the critical point, Physica 48, 541 (1970).

[68] B. Widom, Structure and thermodynamics of interfaces, in U. Landman, Statistical Mechanics and Statistical Methods in Theory and Application (Plenum, New York, 1977).

[69] A. Belhaj, M. Chabab, H. El Moumni, and M. B. Sedra, On thermodynamics of AdS black holes in arbitrary dimensions, Chin. Phys. Lett. 29, 100401 (2012). 